\title{
PERMUTUTATIONAL LABELLING OF CONSTANT WEIGHT GRAY CODES
}

\author{
INESSA LeVI AND Steve SEIF
}

We prove that for positive integers $n$ and $r$ satisfying $1<r<n$, with the single exception of $n=4$ and $r=2$, there exists a constant weight Gray code of $r$-sets of $X_{n}=\{1,2, \ldots, n\}$ that admits an orthogonal labelling by distinct partitions, with each subsequent partition obtained from the previous one by an application of a permutation of the underlying set. Specifically, an $r$-set $A$ and a partition $\pi$ of $X_{n}$ are said to be orthogonal if every class of $\pi$ meets $A$ in exactly one element. We prove that for all $n$ and $r$ as stated, and $i=1,2, \ldots,\left(\begin{array}{l}n \\ r\end{array}\right)$ taken modulo $\left(\begin{array}{l}n \\ r\end{array}\right)$, there exists a list $A_{1}, A_{2}, \ldots, A_{\left(\begin{array}{c}n \\ r\end{array}\right)}$ of the distinct $r$-sets of $X_{n}$ with $\left|A_{i} \cap A_{i+1}\right|=r-1$ and a list of distinct partitions $\pi_{1}, \pi_{2}, \ldots, \pi_{\left(\begin{array}{c}n \\ r\end{array}\right)}$ such that $\pi_{i}$ is orthogonal to both $A_{i}$ and $A_{i+1}$, and $\pi_{i+1}=\pi_{i} \lambda_{i}$ for a suitable permutation $\lambda_{i}$ of $X_{n}$.

\section{ORThogonally LABELled Hamiltonian CYCLES}

We prove a combinatorial result regarding labelling of constant weight Gray codes. The paper is aimed at understanding the combinatorics of subsets and partitions of finite sets and their efficient listing.

Let $X_{n}=\{1,2 \ldots, n\}$. An $r$ element subset $A$ of $X_{n}$ is referred to as an $r$-set. Let $G_{n, r}$ be the graph whose vertices constitute all the $r$-sets of $X_{n}$, with two $r$-sets being adjacent if their intersection has exactly $r-1$ elements. A path in a graph is a sequence of distinct pairwise adjacent vertices; a cycle is a path in which the first and the last vertices are adjacent. A Hamiltonian path (cycle) is one that contains every vertex of the graph. It is well-known that $G_{n, r}$ is Hamiltonian; that is, that it contains Hamiltonian cycles. Hamiltonian cycles of $G_{n, r}$ are also known as constant weight Gray codes and were among the earliest examples of combinatorial Gray codes ([6]).

A partition $\pi$ of $X_{n}$ is said to have weight $r$ if $\pi$ has $r$ distinct classes. The partition $\pi$ and the set $A$ are said to be orthogonal if every class of $\pi$ contains exactly one element of $A$. An orthogonally labelled list of $r$-sets in $X_{n}$ is a sequence

$$
A_{1}, \pi_{1}, A_{2}, \pi_{2}, \ldots, A_{\left(\begin{array}{c}
n \\
r
\end{array}\right)}, \pi_{\left(\begin{array}{c}
n \\
r
\end{array}\right)}
$$

Received 30th August, 2001

Copyright Clearance Centre, Inc. Serial-fee code: 0004-9727/02 \$A2.00+0.00. 
alternating between distinct $r$-sets $A_{i}$ and distinct partitions $\pi_{i}$ of weight $r$, such that for $i=1,2, \ldots,\left(\begin{array}{l}n \\ r\end{array}\right)$ taken modulo $\left(\begin{array}{l}n \\ r\end{array}\right)$, the partition $\pi_{i}$ is simultaneously orthogonal to $A_{i}$ and $A_{i+1}$. The sequence $A_{1}, A_{2}, \ldots, A_{\left(\begin{array}{c}n \\ r\end{array}\right)}$ of $\left(\begin{array}{c}n \\ r\end{array}\right)$ distinct $r$-sets of $X_{n}$ is referred to as the set-sequence, and is denoted by $\mathcal{A}=A_{1} A_{2} \ldots A_{\left(\begin{array}{c}n \\ r\end{array}\right)}$. The sequence $\pi_{1}, \pi_{2}, \ldots, \pi_{\left(\begin{array}{l}n \\ r\end{array}\right)}$ of $\left(\begin{array}{l}n \\ r\end{array}\right)$ distinct partitions is referred to as the partition-sequence, and is denoted by II $=\pi_{1} \pi_{2} \ldots \pi_{\left(\begin{array}{c}n \\ r\end{array}\right)}$. We identify the orthogonally labelled list in (1) with an ordered pair $(\mathcal{A}, \Pi)$. In the sequel, we omit the commas between the elements of set-sequences and partition-sequences.

In [1], Howie and McFadden prove the existence of orthogonally labelled lists as stated below.

THEOREM 1.1. ([1]) For all positive integers $n$ and $r$ with $1<r<n$ there exist an orthogonally labelled list of the r-sets of $X_{n}$.

If the partition-sequence $\Pi$ is such that for each $i=1,2, \ldots,\left(\begin{array}{l}n \\ r\end{array}\right)$ taken modulo $\left(\begin{array}{l}n \\ r\end{array}\right)$, there exists a permutation $\lambda_{i}$ of $X_{n}$ with $\pi_{i+1}=\pi_{i} \lambda_{i}$, the orthogonally labelled list $(\mathcal{A}, \Pi)$ is referred to as the permutational orthogonally labelled list. If the set sequence $\mathcal{A}$ is a Hamiltonian cycle in $G_{n, r}$, the orthogonally labelled list $(\mathcal{A}, \Pi)$ is referred to as an orthogonally labelled Hamiltonian cycle. Our objective in this paper is to prove the following strengthening of Theorem 1.1.

THEOREM 1.2. For all positive integers $n$ and $r$ with $1<r<n$, except for the $n=4, r=2$ case, there exists a permutational orthogonally labelled Hamiltonian cycle in $G_{n, r}$.

We prove the theorem after providing a definition and several examples. A partition of the set $X_{n}$ has type $\tau=d_{1}{ }^{t_{1}} d_{2}{ }^{t_{2}} \ldots d_{k}{ }^{t_{k}}$ if it has $t_{i}$ classes of size $d_{i}$ for $i=1,2, \ldots, k$, where $d_{1}>d_{2} \ldots>d_{k}$. We use $\tau$ to refer to the set of all partitions of $X_{n}$ of that type.

EXAMPLE 1.1. We show that $G_{4,2}$ has no permutational orthogonally labelled Hamiltonian cycle (nor even a permutational orthogonally labelled list). There are seven partitions of weight two of $X_{4}$ : three of these are of type $2^{2}$ and four of type 31 . There are six 2-sets in $X_{4}$; hence, any orthogonally labelled Hamiltonian list in $G_{4,2}$ must contain partitions of both types, $2^{2}$ and 31 . No permutation of $X_{4}$ can transform a partition of one type into the other; hence there exists no permutational orthogonally labelled list in $G_{4,2}$. It is somewhat surprising that $n=4, r=2$ turns out to be the only exceptional case as Theorem 1.2 indicates.

In the table below, we also present two permutational orthogonally labelled Hamiltonian cycles for $n=5$, one for the case of $r=2$, the other for the case $r=3$. 


\begin{tabular}{|c|c|||c|c|}
\hline Set & Partition & Set & Partition \\
\hline$A_{i}$ & $\pi_{i}$ & $B_{i}$ & $\gamma_{i}$ \\
\hline 12 & $25 \mid 134$ & 123 & $3|15| 24$ \\
23 & $12 \mid 345$ & 134 & $3|12| 45$ \\
13 & $14 \mid 235$ & 234 & $2|13| 45$ \\
34 & $23 \mid 145$ & 124 & $4|13| 25$ \\
24 & $34 \mid 125$ & 145 & $4|12| 35$ \\
14 & $24 \mid 135$ & 245 & $4|23| 15$ \\
45 & $15 \mid 234$ & 345 & $3|14| 25$ \\
35 & $45 \mid 123$ & 135 & $5|12| 34$ \\
25 & $35 \mid 124$ & 235 & $5|13| 24$ \\
15 & $13 \mid 245$ & 125 & $1|24| 35$ \\
\hline
\end{tabular}

Figure 1: Permuational Orthogonally Labelled Hamiltonian cycles in $G_{5,2}$ and $G_{5,3}$

An orthogonally labelled list $(\mathcal{A}, \Pi)$ in which every partition in $\Pi$ has type $\tau$, is called an orthogonally $\tau$-labelled list. If $\mathcal{A}$ is a Hamiltonian cycle, then $(\mathcal{A}, \Pi)$ is referred to as an orthogonally $\tau$-labelled Hamiltonian cycle. For a fixed type $\tau$, the group $S_{n}$ of permutations of $X_{n}$ acts transitively on the set of partitions of type $\tau$. In particular, an orthogonally $\tau$-labelled list is a permutational orthogonally labelled list. The following proposition is concerned with the case of partitions of weight two and begins the proof of the theorem.

Proposition 1.3. Let $d \geqslant 3$ and $\tau=d 2$. There exists an orthogonally $\tau$-labelled Hamiltonian cycle in $G_{d+2,2}$.

Proof: We prove inductively that for $d \geqslant 3$ there exists an orthogonally ( $d 2)$ labelled Hamiltonian cycle $(\mathcal{A}, \Pi)$, such that the first set in the set-sequence is $\{1,2\}$, the last set in the set-sequence is $\{1, d+2\}$, and the last partition in the partition sequence has a doubleton class $\{1,3\}$.

The base step with $d=3$ is presented in the two left-most columns of Figure 1; they comprise an orthogonally labelled Hamiltonian cycle in $G_{5,2}$ with the properties described above.

Suppose that for $d \geqslant 4$ there exists an orthogonally $((d-1) 2)$-labelled Hamiltonian cycle $(\mathcal{B}, \Gamma)$, satisfying the above inductive assumptions. Specifically, if $\mathcal{B}=B_{1} B_{2} \ldots B_{\left(\begin{array}{c}d+1 \\ 2\end{array}\right)}$ then $B_{1}=\{1,2\}, B_{\left(\begin{array}{c}d+1 \\ 2\end{array}\right)}=\{1, d+1\}$, and if $\Gamma=\gamma_{1} \gamma_{2} \ldots \gamma_{\left(\begin{array}{c}d+1 \\ 2\end{array}\right)}$ then the doubleton class of $\gamma_{\left(\begin{array}{c}d+1 \\ 2\end{array}\right)}$ is $\{1,3\}$. Then the partition sequence $\Gamma^{\prime}=\gamma_{1}^{\prime} \gamma_{2}^{\prime} \ldots \gamma_{\left(\begin{array}{c}d+1 \\ 2\end{array}\right)}^{\prime}$, obtained from $\Gamma$ by adjoining $d+2$ to the $(d-1)$-class of each partition $\gamma_{i}$ in $\Gamma$, orthogonally labels the cycle $\mathcal{B}=B_{1} B_{2} \ldots B_{\left(\begin{array}{c}d+1 \\ 2\end{array}\right)}$ in $G_{d+2,2}$.

For $i=1,2, \ldots, d+1$, let $C_{i}=\{d+2-i, d+2\}$. Then

$$
\mathcal{A}=B_{1} B_{2} \ldots B_{\left(\begin{array}{c}
d+1 \\
2
\end{array}\right)} C_{1} C_{2} \ldots C_{d+1}
$$


is a Hamiltonian cycle in $G_{d+2,2}$ with $B_{1}=\{1,2\}$ and $C_{d+1}=\{1, d+2\}$. To label $\mathcal{A}$ with orthogonal partitions of type $d 2$, define the following partitions of $X_{d+2}$ : $\pi_{1}=\{1, d+\cdot 2\}\left|\left(X_{d+1}-\{1\}\right), \pi_{2}=\{2, d+2\}\right|\left(X_{d+1}-\{2\}\right)$, and for $i=3,4, \ldots, d+1$, $\pi_{i}=\{d+4-i, d+2\} \mid\left(X_{d+1}-\{d+4-i\}\right)$ (note that for $i=1,2, \ldots, d+1$ the partitions $\pi_{i}$ have $d+2$ in a two element class, and so they are distinct from partitions in $\Gamma^{\prime}$ ). Let $\Pi=\gamma_{1}^{\prime} \gamma_{2}^{\prime} \ldots \gamma_{\left(\begin{array}{c}d+1 \\ 2\end{array}\right)-1}^{\prime} \pi_{1} \pi_{2} \pi_{3} \ldots \pi_{d+1} \gamma_{\left(\begin{array}{c}d+1 \\ 2\end{array}\right)}^{\prime}$, then $(\mathcal{A}, \Pi)$ is an orthogonally $(d 2)$-labelled Hamiltonian cycle in $G_{d+2,2}$ with the doubleton class of $\gamma_{\left(\begin{array}{c}d+1 \\ 2\end{array}\right)}^{\prime}$ being of the form $\{1,3\}$. D

Given a partition type $\tau$ on $X_{n}$, let $\tau \oplus 1$ denote a partition type on $X_{n+1}$ obtained from $\tau$ by adjoining one singleton class. If $\tau$ has a class of size $d_{s}>1$, let $\tau-d_{s}$ be a partition type on $X_{n-1}$ obtained from $\tau$ by reducing the size of one of its $d_{s}$-blocks by 1 .

PROPOSITION 1.4. Let $\tau=d_{1}{ }^{t_{1}} d_{2}{ }^{t_{2}} \ldots d_{k}{ }^{t_{k}}$ be a partition type on $X_{n}$ of weight $r$ having at least two distinct class sizes $d_{s}, d_{t} \geqslant 2$. Suppose that there exist Hamiltonian cycles in $G_{n, r}$ and $G_{n, r+1}$ that can be labelled by partitions of type $\tau$ and $\omega=\left(\tau-d_{s}\right) \oplus 1$ respectively. Then there exists a Hamiltonian cycle in $G_{n+1, r+1}$ that can be labelled by partitions of type $\tau \oplus 1$.

ProOF: Observe that $\omega$ is a partition type on $X_{n}$ of weight $r+1$. Let $\mathcal{A}=A_{1} A_{2} \ldots A_{\left(\begin{array}{c}n \\ r+1\end{array}\right)}$ be a Hamiltonian cycle in $G_{n, r+1}$, and let $\Omega=\sigma_{1} \sigma_{2} \ldots \sigma_{\left(\begin{array}{c}n \\ r+1\end{array}\right)}$ be a corresponding partition sequence of partitions of type $\omega$ that orthogonally labels the cycle. For each partition $\sigma_{i}$ in $\Omega$, let $\sigma_{i}^{\prime}$ be a partition of $X_{n+1}$ of type $\tau \oplus 1$ obtained from $\sigma_{i}$ by adjoining the element $n+1$ to a $\left(d_{s}-1\right)$-class.

Let $\mathcal{B}=B_{1} B_{2} \ldots B_{\left(\begin{array}{c}n \\ r\end{array}\right)}$ be a Hamiltonian cycle in $G_{n, r}$ and let $\Gamma=\gamma_{1} \gamma_{2} \ldots \gamma_{\left(\begin{array}{c}n \\ r\end{array}\right)}$ be a corresponding partition sequence of partitions of type $\tau$ that orthogonally label the cycle. For each $B_{i}$ in $\mathcal{B}$, let $B_{i}^{\prime}$ be the $(r+1)$-set $B_{i} \cup\{n+1\}$. For each partition $\gamma_{i}$ in $\Gamma$, let $\gamma_{i}^{\prime}$ be a partition of $X_{n+1}$ of type $\tau \oplus 1$ obtained from $\gamma_{i}$ by adjoining a new class $\{n+1\}$.

Without loss of generality we may assume that $A_{1}=\{1,2, \ldots, r, r+1\}, A_{\left(\begin{array}{c}n \\ r+1\end{array}\right)}$ $=\{1,2, \ldots, r, n\}$ and $B_{1}^{\prime}=\{1,2, \ldots, r, n+1\}$ and $B_{\left(\begin{array}{c}n \\ r\end{array}\right)}^{\prime}=\{1,2, \ldots, r-1, n, n+1\}$ (or else we simply can relabel the elements of $X_{n}$ ). Choose two partitions of $X_{n+1}$ of type $\tau \oplus 1$ containing $n+1$ in a class of size $d_{t}$ such that $\beta$ is orthogonal to $B_{1}^{\prime}$ and $A_{1}$, and $\delta$ is orthogonal to $A_{\left(\begin{array}{c}n \\ r+1\end{array}\right)}$ and $B_{\left(\begin{array}{c}n \\ r\end{array}\right)}^{\prime}$.

Then $A_{1} A_{2} \ldots A_{\left(\begin{array}{c}n \\ r+1\end{array}\right)} B_{\left(\begin{array}{c}n \\ r\end{array}\right)}^{\prime} \ldots B_{2}^{\prime} B_{1}^{\prime}$ is a Hamiltonian cycle in $G_{n+1, r+1}$ which is $\tau \oplus 1$ labelled by partitions in the sequence $\sigma_{1}^{\prime} \sigma_{2}^{\prime} \ldots \sigma_{\left(\begin{array}{c}n \\ r+1\end{array}\right)-1}^{\prime} \delta \gamma_{\left(\begin{array}{c}n \\ r\end{array}\right)-1}^{\prime} \ldots \gamma_{2}^{\prime} \gamma_{1}^{\prime} \beta$. The partitions in this sequence are distinct, as partitions $\sigma_{i}^{\prime}$ contain the element $n+1$ in a $d_{s}$-class, partitions $\gamma_{i}^{\prime}$ contain $n+1$ in a singleton class, and $\beta, \delta$ contain $n+1$ in a $d_{t}$-class.

The following theorem appears in [2].

THEOREM 1.5. For $r \geqslant 2$ and $1 \leqslant s<r$, there exist orthgonally $2^{s} 1^{r-s}$-labelled Hamiltonian cycles in $G_{s+r, r}$.

So that the work here is self-contained, we prove the aspects of Theorem 1.5 that 
will be used to prove the main theorem (Theorem 1.2).

LEMma 1.6. For $r \geqslant 2$, there exist orthogonally $21^{r-1}$ and $2^{2} 1^{r-2}$-labelled Hamiltonian cycles.

Proof: We prove the existence of stated Hamiltonian cycles with an additional condition, namely that the first set of the set-sequence is $\{1,2, \ldots, r\}$ and the last set of the set-sequence is $\{1,2, \ldots, r-1, n\}$, where $n=r+1$ for the $21^{r-1}$-labelled cycle, and $n=r+2$ for the $2^{2} 1^{r-2}$-labelled cycle.

Let $\mathcal{A}=A_{1} \ldots A_{r+1}$ be any Hamiltonian cycle in $G_{r+1, r}$ with $A_{1}=\{1,2, \ldots, r\}$ and $A_{r+1}=\{1,2, \ldots, r-1, r+1\}$. Let $\Pi=\pi_{1} \pi_{2} \ldots \pi_{r+1}$ be the sequence of partitions of the type $21^{r-1}$ such that the only non-singleton class of $\pi_{i}$ is the symmetric difference of $A_{i}$ and $A_{i+1}$, where $i=1,2, \ldots, r+1$, calculated $\bmod (r+1)$. Then $(\mathcal{A}, \Pi)$ is an orthogonally $21^{r-1}$-labelled Hamiltonian cycle in $G_{r+1, r}$ satisfying the stated conditions on the first and the last set.

Now we prove inductively that for $r \geqslant 3$ there exists an orthogonally $2^{2} 1^{r-2}$-labelled Hamiltonian cycle $(\mathcal{B}, \Gamma)$ in $G_{r+2, r}$ satisfying the stated conditions on the first and the last set. The base step with $r=3$ is presented in the two right-most columns of Figure 1: they comprise an orthogonally $2^{2} 1$-labelled Hamiltonian cycle in $G_{5,3}$ such that the first set is $\{1,2,3\}$ and the last set is $\{1,2,5\}$.

Suppose that for $r \geqslant 4$ there exists an orthogonally $2^{2} 1^{r-3}$-labelled Hamiltonian cycle $(\mathcal{C}, \Psi)$ with the partition-sequence $\mathcal{C}=C_{1} C_{2} \ldots C_{\left(\begin{array}{c}r+1 \\ r-1\end{array}\right)}$ satisfying the following conditions: $C_{1}=\{1,2, \ldots, r-1\}$ and $C_{\left(\begin{array}{c}r+1 \\ r-1\end{array}\right)}=\{1,2, \ldots, r-2, r+1\}$. Note that $\mathcal{C}$ is a Hamiltonian cycle in $G_{r+1, r-1}$, and for each $C_{i}$ in $\mathcal{C}$ let $C_{i}^{\prime}=C_{i} \cup\{r+2\}$ be an $r$-set in $X_{r+2}$. For each partition $\psi_{i}$ in $\Psi$ let $\psi_{i}^{\prime}$ be a partition of weight $r$ of $X_{r+2}$ obtained from $\psi_{i}$ by adjoining a new singleton class $\{r+2\}$. Then the partition sequence $\Psi^{\prime}=\psi_{1}^{\prime} \psi_{2}^{\prime} \ldots \psi_{\left(\begin{array}{c}r+1 \\ r-1\end{array}\right)}^{\prime}$ orthogonally labels the cycle $\mathcal{C}=C_{1}^{\prime} C_{2}^{\prime} \ldots C_{\left(\begin{array}{c}r+1 \\ r+1\end{array}\right)}^{\prime}$ in $G_{r+2, r}$.

By the first paragraph of this proof, there exists an orthogonally $21^{r-1}$-labelled Hamiltonian cycle $(\mathcal{A}, \Pi)$ in $G_{r+1, r}$ with the partition-sequence $\mathcal{A}=A_{1} A_{2} \ldots A_{r+1}$ satisfying the following conditions: $A_{1}=\{1,2, \ldots, r\}$ and $A_{r+1}=\{1,2, \ldots, r-1, r+1\}$. For each partition $\pi_{i}$ in $\Pi$ let $\pi_{i}^{\prime}$ be a partition of the type $2^{2} 1^{r-2}$ of $X_{r+2}$ obtained from $\pi_{i}$ by adjoining the element $r+2$ to a singleton class of $\pi_{i}$ not of the form $\{r-1\}$ or $\{r\}$ (such a singleton class may be selected since $r \geqslant 4$, so each $\pi_{i}$ has at least three singleton classes). Then the partition sequence $\Pi^{\prime}=\pi_{1}^{\prime} \pi_{2}^{\prime} \ldots \pi_{r+1}^{\prime}$ orthogonally labels the cycle $\mathcal{A}$ in $G_{r+2, r}$.

Observe that

$$
\mathcal{B}=A_{1} A_{2} \ldots A_{r+1} C_{\left(\begin{array}{c}
r+1 \\
r-1
\end{array}\right)}^{\prime+1} \ldots C_{2}^{\prime} C_{1}^{\prime}
$$

is a Hamiltonian cycle in $G_{r+2, r}$ with the first set $A_{1}=\{1,2, \ldots, r\}$ and the last set $C_{1}^{\prime}=\{1,2, \ldots, r-1, r+2\}$. Let $\alpha$ be any partition of the type $2^{2} 1^{r-2}$ which is simultaneously orthogonal to $A_{r+1}$ and $C_{\left(\begin{array}{c}r+1 \\ r-1\end{array}\right)}^{\prime}$. Such $\alpha$ has a doubleton class $\{r-1, r+2\}$, 
and so it is not an element of either $\Psi^{\prime}$ or $\Pi^{\prime}$. Let $\beta$ be any partition of the type $2^{2} 1^{r-2}$ simultaneously orthogonal to $C_{1}^{\prime}$ and $A_{1}$. Such a $\beta$ has a doubleton class $\{r, r+2\}$, and so it is also not an element of either $\Psi^{\prime}$ or $\Pi^{\prime}$. Since $\Psi^{\prime}$ or $\Pi^{\prime}$ have no elements in common, the sequence

$$
\Gamma=\pi_{1}^{\prime} \pi_{2}^{\prime} \ldots \pi_{r}^{\prime} \alpha \psi_{\left(\begin{array}{c}
r+1 \\
r-1
\end{array}\right)-1}^{\prime} \ldots \psi_{2}^{\prime} \psi_{1}^{\prime} \beta
$$

consists of distinct partitions of type $2^{2} 1^{r-2}$, and $(\mathcal{B}, \Gamma)$ is an orthogonally $2^{2} 1^{r-2}$-labelled Hamiltonian cycle in $G_{r+2, r}$ satisfying the stated conditions on the first and the last set.

The result below follows from Proposition 1.3, Proposition 1.4, and Lemma 1.6.

Corollary 1.7 .

1. For $n \geqslant 5, d \geqslant 2$ and $r \geqslant 2$, there exists an orthogonally $d 21^{r-2}$-labelled Hamiltonian cycle in $G_{n, r}$.

2. There exist orthogonally 21 and $21^{2}$ labelled Hamiltonian cycles in $G_{3,2}$ and $G_{4,3}$ respectively.

Proof OF THEOREM 1.2: Let $n$ and $r$ be positive integers with $2 \leqslant r<n$, such that $n \neq 4$ if $r=2$. Using Corollary 1.7, we show that there exists a Hamiltonian cycle in $G_{n, r}$ orthogonally labelled by partitions of a given fixed type $\tau$.

If $n \geqslant 3$ and $r=n-1$ and we let $\tau=21^{r-1}$. This allows us to assume that $n \geqslant 5$ and $2 \leqslant r \leqslant n-2$. If $r=2$ let $\tau=(n-2) 2$. If $r=n-2$ let $\tau=2^{2} 1^{r-2}$. If $2<r<n-2$ we let $\tau=d 21^{r-2}$, where $d \geqslant 3$.

1.1. Hamiltonian CyCles $H_{n, r}$. For given $n$ and $r$ with $1 \leqslant r<n$, we present the definition of the Hamiltonian cycle $H_{n, r}$. The cycles $H_{n, r}$ arise in the context of reflected Gray codes, certain widely studied recursively defined codes that list the subsets of $X_{n}$ so that successive sets have a singleton symmetric difference. Numerous algorithms for the efficient output of $H_{n, r}$ appear in the literature $([7,5,8])$. Below we shall outline an argument that supports the following refinement of Theorem 1.2.

THEOREM 1.8. For all positive integers $n$ and $r$ with $1<r<n$, except for the $n=4, r=2$ case, there exists a permutational orthogonally labelled Hamiltonian cycle in $G_{n, r}$ with set-sequence $H_{n, r}$.

DEFINITION 1.9: Let $n, r$ be positive integers with $r \leqslant n$, and let $H_{n, r}$ be defined recursively as follows:

1. $H_{n, n}=X_{n}$.

2. $H_{n, 1}=\{1\} \ldots\{n\}$.

3. For $1<r<n$, given that $H_{n-1, r-1}=A_{1} A_{2} \ldots A_{\left(\begin{array}{c}n-1 \\ r-1\end{array}\right)}$, let $H_{n-1, r-1}^{r e v} \oplus n$ be the list

$$
\left(A_{\left(\begin{array}{c}
n-1 \\
r-1
\end{array}\right)} \cup\{n\}\right) \ldots\left(A_{2} \cup\{n\}\right)\left(A_{1} \cup\{n\}\right),
$$

that results by adjoining $n$ to each set of $H_{n-1, r-1}$ and then reversing the order of the resulting listing. 
4. For $1<r<n$, let $H_{n, r}=H_{n-1, r}\left(H_{n-1, r-1}^{r e v} \oplus n\right)$ be the list that results from concatenating $H_{n-1, r}$ and $H_{n-1, r-1}^{r e v} \oplus n$.

ExAmPle 1.2.

$$
\begin{aligned}
& H_{3,2}=H_{2,2}\left(H_{2,1}^{r e v} \oplus 3\right)=\{12\}\{23\}\{13\} \\
& H_{4,2}=H_{3,2}\left(H_{3,1}^{r e v} \oplus 4\right)=\{12\}\{23\}\{13\}\{34\}\{24\}\{14\} \\
& H_{4,3}=\{123\}\{134\}\{234\}\{124\} \\
& H_{5,3}=H_{4,3}\left(H_{4,2}^{r e v} \oplus 5\right)=\{123\}\{134\}\{234\}\{124\}\{145\}\{245\}\{345\}\{135\}\{235\}\{125\} .
\end{aligned}
$$

Notice that the base step of the inductive proof of Proposition 1.3 involves the cycle $H_{5,2}$. The inductive procedure used to $(d 2)$-label Hamiltonian cycles in Proposition 1.3 leads to set-sequences which are $H_{d+2,2}$ cycles. The construction used in Proposition 1.4 guarantees that if the two given cycles are $H_{n-1, r}$ and $H_{n, r+1}$, then the resulting $\tau \oplus 1$ labelled cycle is $H_{n+1, r+1}$. Thus, we may assume that for $d \geqslant 3$, the $\left(d 21^{r-2}\right)$-labelled Hamiltonian cycles used in the proof of Theorem 1.2 are all $H_{n, r}$ cycles.

The Hamiltonian cycle in $G_{5,3}$ in Figure 1 is $H_{5,3}$. We can assume the Hamiltonian cycles of $G_{r+1, r}$ used in the proof in Lemma 1.6 are $H_{r+1, r}$ cycles. Once again, the inductive procedure used in the proof of Lemma 1.6 leads to $H_{r+2, r}$ cycles for $2^{2} 1^{r-2}$ cases. Thus we may assume that all the orthogonally labelled cycles in Corollary 1.7 are $H_{n, r}$ cycles. Theorem 1.8 follows.

\section{Conclusion}

In this work the improvement over existing literature involves the "permutational" aspect of our main theorem. Indeed in [3], the present authors and R. B. McFadden prove that for any Hamiltonian cycle $\mathcal{A}$ there exists a partition sequence $\Pi$ such that $(\mathcal{A}, \Pi)$ is an orthogonally labelled Hamiltonian cycle. They provide a highly efficient algorithm that on input $(n, r)$ outputs an orthogonally labelled Hamiltonian cycle. However, except for the $(3,2)$ case, the partition sequence associated with their algorithm is not permutational. In [3] the Transposition Listing Conjecture is stated: for $n \geqslant 2 r$, the authors conjecture that there exists a permutational orthogonally labelled Hamiltonian cycle such that all permutations involved are transpositions. The authors show that the validity of the Transposition Listing Conjecture is a logical consequence of the celebrated Middle Levels Conjecture (for a reference on the Middle Levels Conjecture, see [6]).

The partition type $\tau$ is said to be exceptional ([2]) if the number of distinct partitions of type $\tau$ is less than $\left(\begin{array}{l}n \\ r\end{array}\right)$. Clearly if $\tau$ is an exceptional partition type, no orthogonally $\tau$-labelled list exists. In [2], the first author and J. Lehel prove existence of orthogonally $\tau$-labelled lists for all non-exceptional partition types $\tau$ with classes of size at most two, a result we used in the paper. Moreover they show that for $1 \leqslant s<r$, there exist 
orthogonally $2^{s} 1^{r-s}$-labelled Hamiltonian cycles. In [4], the authors extend this result and show that even for non-exceptional $\tau$ of the form $2^{r}$, there exist orthogonally $2^{r}$ labelled Hamiltonian cycles.

In [2] it is conjectured that for every non-exceptional type $\tau$, there exists orthogonally $\tau$-labelled list. The present paper is a part of a series of papers directed towards proving this conjecture.

\section{REFERENCES}

[1] J.M. Howie and R.B. McFadden, 'Idempotent rank in finite full transformation semigroups', Proc. Royal Soc. Edinburgh 114 (1990), 161-167.

[2] J. Lehel and I. Levi, 'Loops with partitions and matchings', Ars Combin. 54 (2000), $237-253$.

[3] I. Levi, R.B. McFadden and S.Seif, 'Algorithms for labeling Gray codes', (submitted).

[4] I. Levi and S. Seif, 'Constant weight Gray codes labeled by partitions with blocks of size at most two', Ars Combin. (to appear).

[5] E.M. Reingold, J. Nievergelt and N. Deo, Combinatorial algorithms, theory and practice (Prentice-Hall, Englewood Cliffs, NJ, 1977).

[6] C.D. Savage and P. Winkler, 'Monotone Gray codes and the middle levels problem', $J$. Combin. Theory Ser. A 70 (1995), 230-248.

[7] H.S. Wilf, Combinatorial algorithms: an update, CBMS-NSF Regional Conference Series in Applied Mathematics 55 (SIAM, Philadelphia, PA, 1989).

[8] A.J. van Zanten, 'Index system and separability of constant weight Gray codes', IEEE Trans. Inform. Theory 37 (1991), 1229-1223.

Department of Mathematics

University of Louisville

Louisville, KY 40292

United States of America 